\title{
IMAGE CLASSIFICATION BY PATTERN AND STRUCTURE FEATURES CLUSTERING
}

\author{
Roman Melnyk, Ruslan Tushnytskyy
}

Lviv Polytechnic National University, 12, S. Bandery str., Lviv, 79013, ramelnyk@polynet.lviv.ua, ruslan.tushnytskyy@gmail.com

\begin{abstract}
Resume: An approach for decomposition of visual images by clustering and pattern classification by structure features is considered. Multilevel hierarchical clusters such as rectangles, closed regions and integrated areas are proposed. Hierarchically constructed fragments are material to form pattern structure features. To reduce the clustering algorithm complexity the tolerance coefficient and quality criteria for merging process are proposed. The results of pattern classification by structure features for some image groups by hand and automatic regimes are presented in the article. Hierarchical trees are got for different number of structure coefficients as well as for absolute and relative merging functions.
\end{abstract}

Keywords: clustering, visual pattern, hierarchical tree, rectangles, closed regions, integrated areas, structure features, classification, hierarchical clustering algorithm, tolerance coefficient, specific density, volume.

\section{INTRODUCTION}

For large database with over tens of thousands of visual patterns, effective indexing becomes an important issue of the content-based image retrieval systems (CBIR). This problem is actual in the modern informative systems. A successful categorization of images will greatly enhance the performance of CBIR systems by filtering out images from irrelevant classes during matching [1]. The internet is an example of the distributed database which contains visual patterns. A search in the up-diffused information base is an intricate problem, one of the going near the decision of which is considered in the work.

CBIR system operate in two phases: indexing and searching. In the indexing phase, each image of the database is represented using a set of image attribute (vector of features). The existent universal CBIR systems attribute to one of three categories depending on approach of extracting features: a histogram, coloured location, and region-based. Such properties in particular, is: color [2-3], shape [4-5], structure [6] and location [7]. Extracted features are stored in a visual feature database. In the searching phase, when a user makes a query, a feature vector for the query is compared to the vectors in the feature database. The images most similar to the query are returned to the user.

Region-based image retrieval systems use local properties of regions (ideally objects) as opposed to the global properties of the entire image. Example of regions-based systems includes SIMPLIcity [8]. If objects within the image are segmented and each object feature is extracted automatically, these feature make possible object-based image retrieval [9]. However, representing of the images by adequate number of clusters (objects in the image) can better present the content of them but this approach is time consuming.

Minka and Picard [11] introduced a learning component in their CBIR system. The system requires the supervised training of various parts of the image. Szummer and Picard [10] have developed a system to classify indoor and outdoor scenes. Classification over low-level image features such as color histogram and discrete cosine transformation (DCT) coefficients is performed. A 90 percent accuracy rate has been reported over database of 1300 images from Kodak.

Other examples of semantic image classification includes comparison work of city and landscape [1] and finding of person [12]. Wang and Fischler [13] shows that exact semantic presentation is useful to the image comparison problem.

Presented work is devoted to investigation of structural properties of image, got by the 3 -stages clustering algorithm. 


\section{VISUAL PATTERN STRUCTURAL CHARACTERISTICS}

Visual pattern we decompose and consider as a set of objects having its own complex structure presented by 3 -stages hierarchical tree [Fig.1]. We call the objects from the highest to the lowest level as: integrated area (its number we define as $I A$ ), closed region $(C R)$, cluster (rectangle, $C$ ), microcluster $(M C)$.

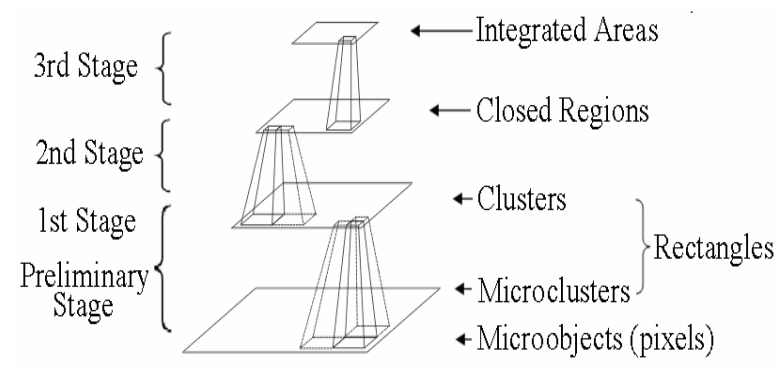

Fig. 1 - Fragment of 3-stages hierarchical tree for pattern

The objects from different stages are characterized by dimension, brightness, topology and figure. Hierarchical structure of objects are reached by decription of them as group of objects from lower stage, i.e. integrated area by closed regions, closed region by clusters (rectangles) and rectangle by microclusters. Fig.2 illustrates the objects to be analized on every stage of the structure tree.

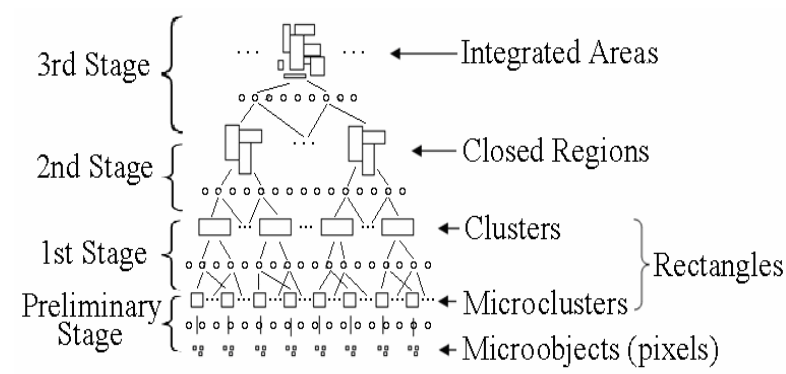

Fig. 2 - Objects of 3-stages hierarchical tree

The coverage of pattern by three types of defined objects $(C, C R, I A)$ are demonstrated on Fig.3.

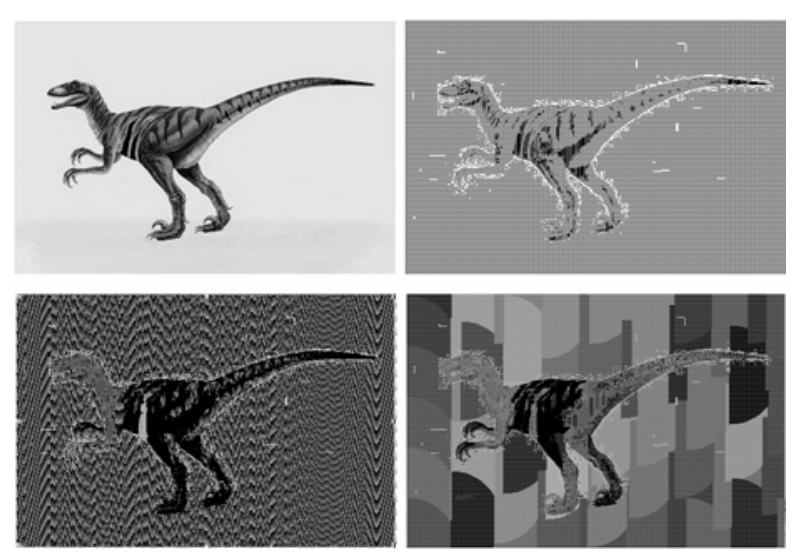

Fig. 3 - Structure objects of 3-stages: original, covered by rectangles, closed regions and integrated areas
On Fig. 3 colours are assigned randomly and by small sizes it is difficult to view the rectangles.

Let us use a number of parameters to denote image structure degree for pattern estimation by its structural properties:

1) structure coefficients indicating transformation degree between two neighbouring levels (an index specifies the level):

$$
K_{s}^{1}=C / M C, K_{s}^{2}=C R / C, K_{s}^{3}=I A / C R
$$

2) structure coefficients indicating transformation degree between two levels throughout one level (indexes specify the levels):

$$
K_{s}^{12}=C R / M C, \quad K_{s}^{23}=I A / C .
$$

3) structure coefficients indicating transformation degree between two levels throughout two levels (indexes specify the levels):

$$
K_{s}^{123}(M C)=I A / M C .
$$

The reverse formulas one could use to estimate fuzziness degree on different structure levels, for example,

$$
K_{f}^{123}(M C)=M C / I A \text {. }
$$

Sometimes, it could be useful the structure coefficient related to all pixels number $P X$, for example:

$$
K_{s}^{123}(P X)=I A / P X .
$$

Each $i$-th selected fragment, in particular, area or region could be characterized by relative number of pixels:

$$
v x_{i}(C R)=p x_{i}(C R) / P X, v x_{i}(I A)=p x_{i}(I A) / P X,
$$

for which inequalities are as follows:

$$
\sum p x_{i}(C R) \leq P X, \quad \sum p x_{i}(I A) \leq P X,
$$

where sums are for all regions and areas.

Some statistical properties could also be considered as structural properties, e.g.:

1) Mean region and area dimension:

$$
\begin{aligned}
M(C R) & =(1 / C R) \cdot \sum p x_{i}(C R), \\
M(I A) & =(1 / I A) \cdot \sum p x_{i}(I A) .
\end{aligned}
$$

2) Region and area deviation:

$$
\begin{aligned}
D(C R) & =\sqrt{(1 / C R) \cdot \sum\left(p x_{i}(C R)-M(C R)\right)^{2}}, \\
D(I A) & =\sqrt{(1 / I A) \cdot \sum\left(p x_{i}(I A)-M(I A)\right)^{2}} .
\end{aligned}
$$

3) To estimate the image elementary object 
size/deviation features on different tree levels we consider propose statistical relations:

$$
R(C R)=\frac{M(C R)}{D(C R)}, \quad R(I A)=\frac{M(I A)}{D(I A)},
$$

and full image integral statistical relations:

$$
R_{I}(C R)=\frac{C R \cdot M(C R)}{D(C R)}, \quad R_{I}(I A)=\frac{I A \cdot M(I A)}{D(I A)} .
$$

So, to characterize an image we could by full set of pattern structure features: $P X, M C, C, C R, I A$, $K_{s}^{1}, K_{s}^{2}, K_{s}^{3}, K_{s}^{12}, K_{s}^{23}, K_{s}^{123}(M C), K_{s}^{123}(P X)$, $M(C R), D(C R), R(C R), R_{I}(C R), M(I A), D(I A), R(I A)$, $R_{I}(I A)$ or a part from them. To find this structure set we use the three stages clustering algorithm [14 16] based on hierarchical rolling-up tree.

\section{CLASSIFICATION BY STRUCTURAL COEFFICIENTS}

To investigate the pattern structure features some examples of images shown on Fig. 4 were estimated by the algorithm.

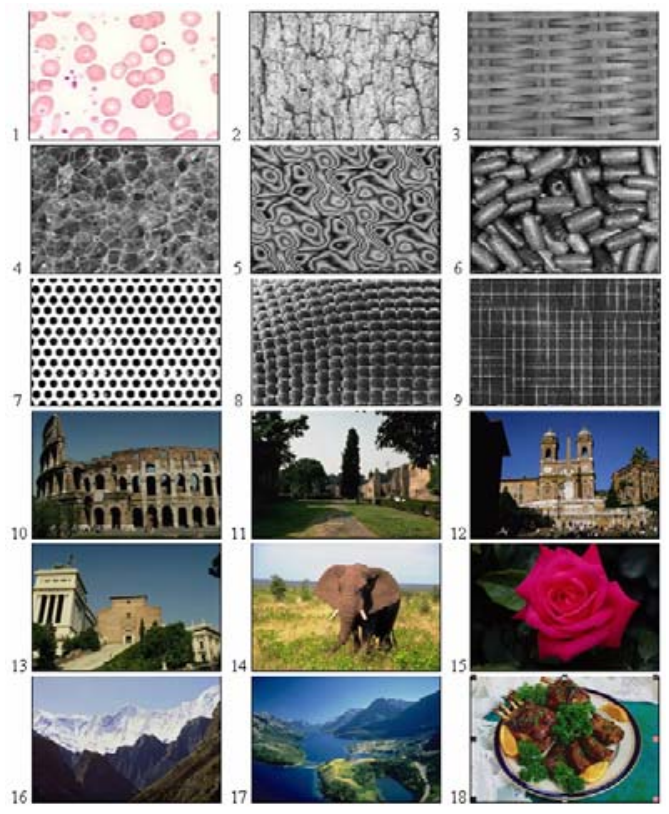

Fig. 4 - Pattern samples

Table 1 contains pattern structure features. At first step a region number $I A$, structure coefficients $K_{s}^{123}(M C)$ and $K_{s}^{123}(P X), M(I A), D(I A)$ were sorted separately. Then, integral pattern classification in the table is got by sorting a sum of pattern places got for $I A, K_{s}^{123}(M C), K_{s}^{123}(P X)$ parameters. This classification is shown on Fig.5.

Choosing brightness cluster parameter we get different image classification. For example, Table 1 presents result for cluster brightness parameter $b=$
$75 \%$, Table $2-b=85 \%$, Table $3-b=100 \%$. Their integral image classification is shown on Fig.5, Fig.6 and Fig. 7 accordingly.

Table 1 confirms possibility to create the classification rules for medical images, material structure, etc. In particular, taking into account the pattern parameters $M(I A)$ and $D(I A)$ on Fig. 5 class $\mathrm{C}$ can be broken up into separate class of blood cells (image № 1) and textures (image № 3,9).

Table 1. Pattern Classification

\begin{tabular}{|c|c|c|c|c|c|c|c|c|c|}
\hline Place & $I A$ & $\begin{array}{c}\operatorname{Img} g \\
M\end{array}$ & $K_{3}^{123}(\mathrm{MC}$ & $\operatorname{Img}$ & $K_{s}^{123}(P N)$ & $\begin{array}{c}\operatorname{Irgg} \\
\mathrm{Ng}\end{array}$ & $M(I A)$ & $(I A)$ & $\begin{array}{l}\text { Integr. } \\
\text { classif. }\end{array}$ \\
\hline 1 & 178 & 2 & 0,00838 & 7 & 0,00548 & 2 & 175,43 & 124,91 & 2 \\
\hline 2 & 170 & 4 & 00735 & 1 & 0523 & 4 & 191,08 & 166,48 & 4 \\
\hline 3 & 148 & 5 & 0,00570 & 2 & 0,00456 & 5 & 219,49 & 177,61 & 7 \\
\hline 4 & 146 & 7 & 1523 & 4 & 0449 & 7 & 119,32 & 244,53 & 5 \\
\hline 5 & 143 & 6 & 0,00456 & 5 & 0,00440 & 6 & 225,80 & 571,13 & 6 \\
\hline 6 & 131 & 14 & 0,00443 & 6 & 0,00403 & 14 & 247,61 & 376,77 & 14 \\
\hline 7 & 130 & 8 & 405 & 8 & 1400 & 8 & 246,79 & 1440,20 & 8 \\
\hline 8 & 126 & 18 & 0,00404 & 14 & 0,00388 & 18 & 257,67 & 1013,51 & 18 \\
\hline 9 & 108 & 9 & 0,00388 & 18 & 0,00332 & 3 & 300,81 & 194,52 & 3 \\
\hline 10 & 108 & 3 & 0,00332 & 3 & 0,00332 & 9 & 300,78 & 1066,08 & 9 \\
\hline 11 & 97 & 10 & 32 & 9 & 199 & 10 & 334,92 & 1098,05 & 1 \\
\hline 12 & 94 & 13 & 0,00299 & 10 & 0,00289 & 13 & 345,61 & 717,35 & 10 \\
\hline 13 & 91 & 12 & 0,00289 & 13 & 0,00280 & 12 & 356,74 & 1067,25 & 13 \\
\hline 14 & 84 & 17 & 0,00280 & 12 & 0,00259 & 17 & 386,75 & 1093,51 & 12 \\
\hline 15 & 83 & 11 & 0,00259 & 17 & 0,00255 & 11 & 388,57 & 1339,15 & 17 \\
\hline 16 & 82 & 1 & 0,00257 & 11 & 0,00252 & 1 & 136,11 & 137,41 & 11 \\
\hline 17 & 63 & 16 & 0,00195 & 16 & 0,00194 & 16 & 513,29 & 2004,53 & 16 \\
\hline 18 & 4 & 15 & 0,00012 & 15 & 0,00012 & 15 & 8121,75 & 14019,37 & 15 \\
\hline
\end{tabular}

by the Structural Characteristics, $b=75 \%$

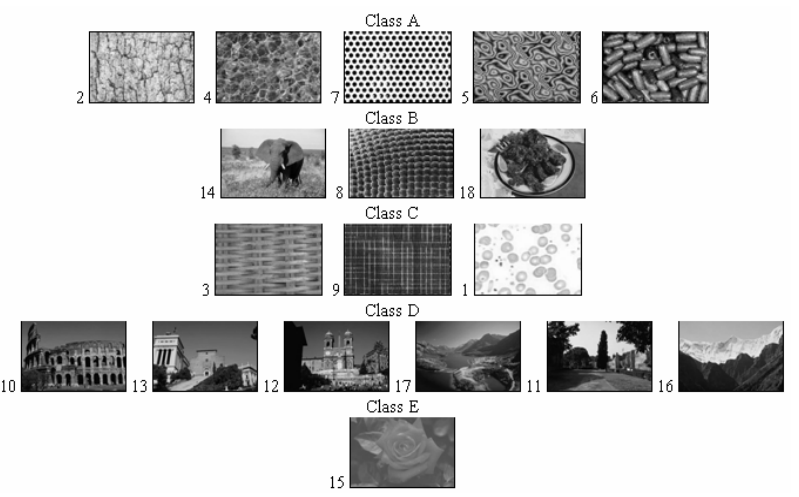

Fig. 5 - Integral pattern classification (for $b=75 \%$ )

Table 2. Pattern Classification

\begin{tabular}{|c|c|c|c|c|c|c|c|c|c|}
\hline Place & $I A$ & $\begin{array}{c}\operatorname{Irng} \\
\mathrm{N} / \mathrm{g}\end{array}$ & $K_{s}^{-123}(M C)$ & $\begin{array}{c}\operatorname{Img} g \\
N \text { ga }\end{array}$ & $K_{s}^{-123}(F I)$ & $\operatorname{ling}$ & $M(I A)$ & $D(I A)$ & $\begin{array}{l}\text { Irtegr. } \\
\text { classif. }\end{array}$ \\
\hline 1 & 193 & 4 & 0,01091 & 7 & 0,00594 & 4 & 168,31 & 140,24 & 4 \\
\hline 2 & 190 & 7 & 0,00735 & 1 & 0,00585 & 7 & 91,69 & 155,23 & 7 \\
\hline 3 & 183 & 2 & 0,00594 & 4 & 0,00563 & 2 & 170,63 & 125,99 & 2 \\
\hline 4 & 175 & 5 & 0,00586 & 2 & 0,00539 & 5 & 185,62 & 92,81 & 5 \\
\hline 5 & 170 & 6 & 0,00539 & 5 & 0,00523 & 6 & 189,94 & 222,73 & 6 \\
\hline 6 & 169 & 8 & 0,00527 & 8 & 0,00520 & 8 & 189,84 & 214,51 & 8 \\
\hline 7 & 162 & 9 & 0,00526 & 6 & 0,00499 & 9 & 200,52 & 220,76 & $\overline{9}$ \\
\hline 8 & 151 & 18 & 0,00499 & 9 & 0,00465 & 18 & 215,01 & 273,49 & 18 \\
\hline 9 & 140 & 14 & 0,00465 & 18 & 0,00431 & 14 & 231,70 & 304,53 & 10 \\
\hline 10 & 123 & 10 & 0,00432 & 14 & 0,00379 & 10 & 264,12 & 319,70 & 11 \\
\hline 11 & 121 & 11 & 0,00379 & 10 & 0,00372 & 11 & 266,54 & 1041,71 & 1 \\
\hline 12 & 118 & 13 & 0,00375 & 11 & 0,00363 & 13 & 275,31 & 347,93 & 14 \\
\hline 13 & 117 & 12 & 0,00363 & 13 & 0,00360 & 12 & 277,46 & 586,27 & 13 \\
\hline 14 & 116 & 17 & 0,00360 & 12 & 0,00357 & 17 & 280,06 & 372,54 & 12 \\
\hline 15 & 114 & 3 & 0,00357 & 17 & 0,00351 & 3 & 284,97 & 198,93 & 17 \\
\hline 16 & 83 & 16 & 0,00351 & 3 & 0,00255 & 16 & 389,60 & 965,76 & 3 \\
\hline 17 & 82 & 1 & 0,00257 & 16 & 0,00252 & 1 & 136,11 & 137,41 & 16 \\
\hline 18 & 28 & 15 & 0,00086 & 15 & 0,00086 & 15 & 1160,25 & 3130,79 & 15 \\
\hline
\end{tabular}
by the Structural Characteristics, $b=85 \%$ 


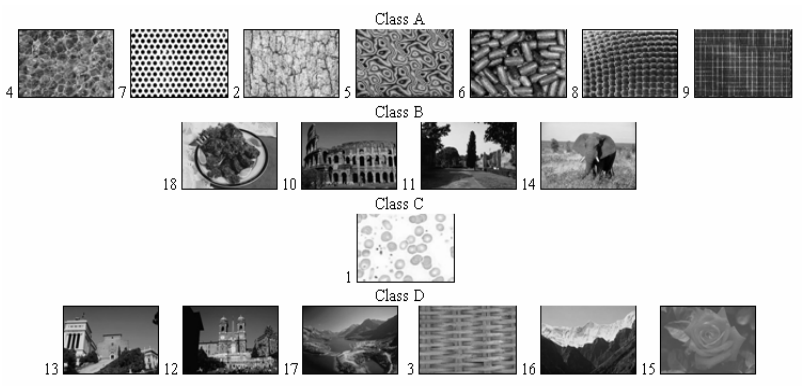

Fig.6 - Integral pattern classification (for $b=85 \%$ )

Table 3. Pattern Classification

by the Structural Characteristics, $b=\mathbf{1 0 0} \%$

\begin{tabular}{|c|c|c|c|c|c|c|c|c|c|}
\hline Place & $I A$ & $\begin{array}{c}\operatorname{Ing} g \\
\mathrm{Ml}\end{array}$ & $K_{s}^{123}(M O$ & $\underset{\mathrm{Irng}}{\operatorname{Irg}}$ & $K_{s}^{123}(P R)$ & $\operatorname{Irng}_{\mathrm{Mg}}$ & $M(I A)$ & $D(I A)$ & $\begin{array}{l}\text { Integr. } \\
\text { classif. }\end{array}$ \\
\hline 1 & 225 & 7 & 0,01292 & 7 & 0,00693 & 7 & 77,43 & 58,89 & 7 \\
\hline 2 & 201 & 6 & 0,00708 & $\overline{1}$ & 0,00619 & 6 & 160,64 & 97,07 & 6 \\
\hline 3 & 198 & 4 & 0,00623 & 6 & 0,00609 & 4 & 164,06 & 139,29 & 4 \\
\hline 4 & 189 & 5 & 0,00610 & 4 & 0,00582 & 5 & 171,87 & 97,88 & 5 \\
\hline 5 & 177 & 2 & 0,00582 & 5 & 0,00545 & 2 & 176,42 & 132,92 & 2 \\
\hline 6 & 175 & 8 & 0,00567 & 2 & 0,00539 & 8 & 183,33 & 151,55 & 8 \\
\hline 7 & 168 & 18 & 0,00545 & 8 & 0,00517 & 18 & 193,26 & 176,10 & 18 \\
\hline 8 & 161 & 11 & 0,00517 & 18 & 0,00496 & 11 & 200,32 & 271,94 & 11 \\
\hline 9 & 160 & 9 & 0,00499 & 11 & 0,00493 & 9 & 203,03 & 236,25 & 9 \\
\hline 10 & 151 & 10 & 0,00493 & 9 & 0,00465 & 10 & 215,15 & 251,15 & 10 \\
\hline 11 & 142 & 14 & 0,00465 & 10 & 0,00437 & 14 & 228,43 & 327,98 & 14 \\
\hline 12 & 140 & 12 & 0,00438 & 14 & 0,00431 & 12 & 231,88 & 456,16 & 1 \\
\hline 13 & 139 & 13 & 0,00431 & 12 & 0,00428 & 13 & 233,72 & 369,20 & 12 \\
\hline 14 & 113 & 17 & 0,00428 & 13 & 0,00348 & 17 & 287,50 & 269,84 & 13 \\
\hline 15 & 109 & 3 & 0,00348 & 17 & 0,00336 & 3 & 298,05 & 218,23 & 17 \\
\hline 16 & 98 & 16 & 0,00336 & 3 & 0,00302 & 16 & 329,97 & 388,39 & 3 \\
\hline 17 & 79 & 1 & 0,00303 & 16 & 0,00243 & 1 & 141,28 & 147,37 & 16 \\
\hline 18 & 70 & 15 & 0,00215 & 15 & 0,00215 & 15 & 464,1 & 466,93 & 15 \\
\hline
\end{tabular}

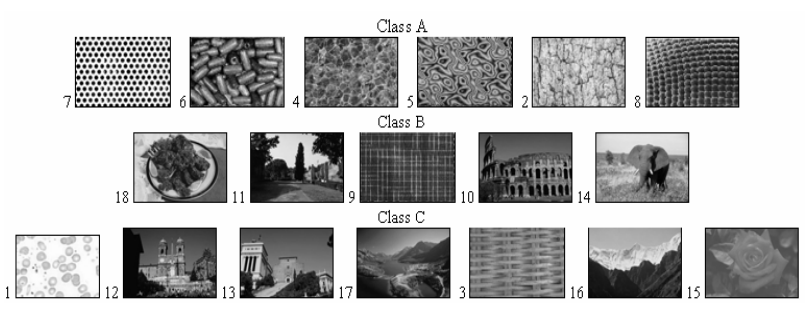

Fig. 7 - Integral pattern classification (for $b=100 \%$ )

Fig. 8 presents classification result got by sorting sum of pattern places, classificated separately by one of $I A, K_{s}^{123}(M C), K_{s}^{123}(P X)$ and $D(I A) / M(I A)$ parameters.
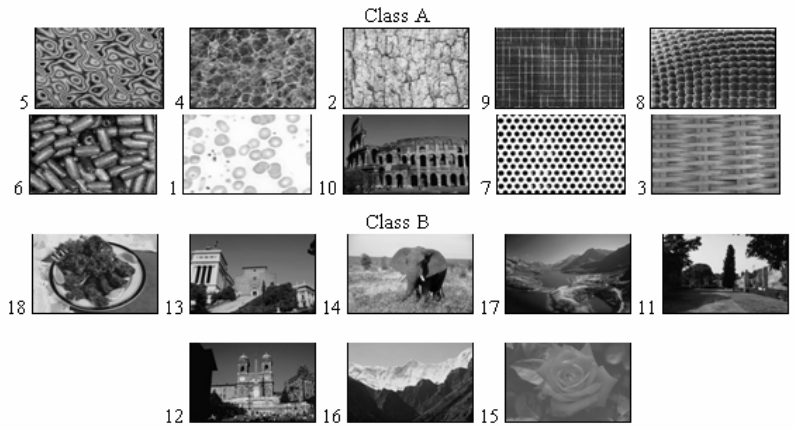

Fig.8 - Integral pattern classification including $\mathrm{D}(\mathrm{IA}) / \mathrm{M}(\mathrm{IA})$ (for $\mathrm{b}=\mathbf{8 5} \%$ )

\section{CLUSTERING ALGORITHM FOR STRUCTURAL CHARACTERISTICS}

Practical classes of data clustering problems are very time-consuming due to the data volumes and algorithm complexity [17-20]. To reduce time losses satisfying the accuracy requirements we propose approach similar but more simple that proposed in [20].

The traditional agglomerative hierarchical algorithm for data clustering has the following steps:

S0. For all points $x_{i}, x_{j} \in X$.

$\mathrm{S} 1$. To find the candidates pairs by calculating the similarity distances, i.e.

$$
\forall\left(x_{i}, x_{j}(j>i)\right) \text { CALCULATE } F\left(x_{i}, x_{j}\right) \text {. }
$$

$\mathrm{S} 2$. To find the pair having the best distance value

$$
F^{*}\left(x_{i}, x_{j}\right)=\min F\left(x_{i}, x_{j}\right), \quad i, j \in I,
$$

and to merge points $x_{i}, x_{j}$, creating a new cluster $x_{n+1}$.

S3. To delete points $x_{i}, x_{j}$ from the candidate list.

S4. The end (for all $x_{i}, x_{j} \in X$ ).

Having the best accuracy the universal hierarchical algorithm is characterized by polynomial complexity $O\left(N^{3}\right)$. To reduce complexity to $O\left(N^{2}\right)$ the authors in [20] propose to find so called friends merged on the same tree level. We should like to note that authors do not explain the costs needed to evaluate simplicity distances for all friends at every tree level.

We propose the similar idea to reduce the algorithm complexity without calculation of friend distances but imposing in the algorithm the distance tolerance at tree level to merge clusters. At the step S3 of the classical algorithm we merge those pairs of points satisfying the following condition:

$$
F\left(x_{i}, x_{j}\right) \geq F_{0} \cdot\left(1-k_{v}\right),
$$

where $F_{0}$ - the best distance value on the merging level, $k_{v}\left(k_{v}<1\right)-$ a tolerance coefficient indicating distances for pairs to be merged on the current tree level (we also can call it as the speed and accuracy coefficient).

Function $F$ is being formed as weighted average linkage by Manhattan distance:

$$
F_{i j}=\left\{w_{1}\left|a_{i}-a_{j}\right|+w_{2}\left|b_{i}-b_{j}\right|+\ldots\right\} / k \cdot r,
$$

or sum of squares distance:

$$
F_{i j}=\left\{w_{1}\left[a_{i}-a_{j}\right]^{2}+w_{2}\left[b_{i}-b_{j}\right]^{2}+\ldots\right\} / N,
$$

where $a, b, c$ are characteristic features (coordinates of the centre, the number of image lines intersections with the coordinate axes, etc.) that create the image key and are expressed in numerical values; $k, r$ are the numbers of points in $i$ and $j$ clusters of the 
rolling-up tree. Summation is taking place by all point values, $N$ - dataset number.

The relative merging function $F$ is being formed as relation between Manhattan distance and a maximum value of two coordinates:

$$
F_{i j}=\left\{\frac{w_{1}\left|a_{i}-a_{j}\right|}{\max \left\{a_{i}, a_{j}\right\}}+\frac{w_{2}\left|b_{i}-b_{j}\right|}{\max \left\{b_{i}, b_{j}\right\}}+\ldots\right\} /(k+r),
$$

or as sum of relative square distances:

$$
F_{i j}=\left\{\frac{w_{1}\left[a_{i}-a_{j}\right]^{2}}{\max \left\{a_{i}, a_{j}\right\}}+\frac{w_{2}\left[b_{i}-b_{j}\right]^{2}}{\max \left\{b_{i}, b_{j}\right\}}+\ldots\right\} /(k+r),
$$

For new cluster its characteristics are being formed as average of two entering cluster characteristics (centroid):

$$
Q_{l}=Q_{l}\left(\left(a_{i}+a_{j}\right) / 2,\left(b_{i}+b_{j}\right) / 2,\left(c_{i}+c_{j}\right) / 2, \ldots\right),
$$

or weighted average of two component characteristics (weighted centroid):

$$
Q_{k}=Q_{k}\left(\left(k \cdot a_{i}+r \cdot a_{j}\right) /(k+r),\left(k \cdot b_{i}+r \cdot b_{j}\right) /(k+r), \ldots\right),
$$

For the quality evaluation of clusters on the different levels of the tree we use the Ward criterion of sum-of-squares deviations:

$$
\begin{gathered}
E=\left\{\sum E_{k}\right\} / M=\left\{\sum \sum d_{j}\right\} / M=\left\{\sum \sum\left(a_{i}^{*}-a_{j}\right)^{2}+\left(b_{i}^{*}-b_{j}\right)^{2}+\ldots . \mid m_{j}\right\} / M, \\
k \in \overline{1, M}, \quad i, j \in J
\end{gathered}
$$

where $a_{i}{ }^{*}, b_{i}{ }^{*}, c_{i}^{*}$ are coordinates of the cluster centre, $a_{i}, b_{i}, c_{i}$ - coordinates of the cluster, $E_{k}-$ cluster deviation, $E$ - integral deviation, $d_{j}-$ deviation of the cluster from the cluster centre, $M-\mathrm{a}$ number of clusters on the controlled level of the hierarchical tree.

To reduce time losses caused by quality function evaluation we propose more simple functions - the space density for a given level of the hierarchical tree:

$$
S=\left\{\sum L_{k} / D_{k}\right\} / M,
$$

where $L_{k}, D_{k}$ are a number of clusters and its diameter.

Close to the density function we also consider volume per item function:

$$
V=\left\{\sum D_{k} / L_{k}\right\} / M
$$

In the equations $(20,21)$ to estimate the cluster space the cluster diameter $D_{k}$ as maximal distance between two clusters is accepted.

First experiments were held to confirm validity of functions to estimate the algorithm accuracy. Fig. 9 illustrates changes in the similarity function (a) and specific dispersion (b) during the merging process for $k_{v}$ coefficient values $0,0.5 \%, 0.75 \%, 1 \%, 5 \%$, $10 \%, 25 \%$ by rolling-up level.

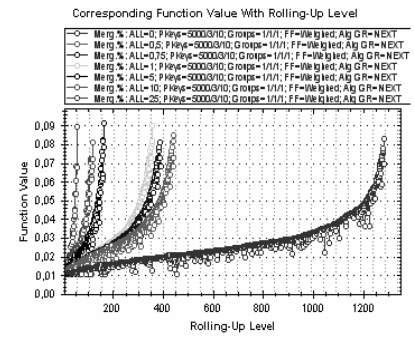

(a)

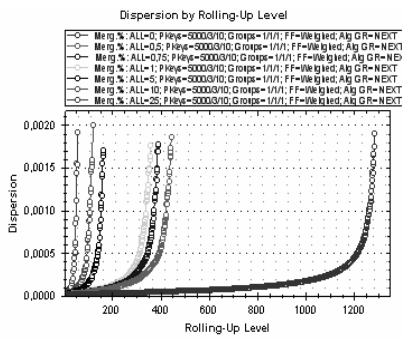

(b)
Fig.9 - Similarity and specific dispersion functions by the rolling-up tree level

Fig.10 illustrates specific capacity (a) and density (b) dependence for $k_{v}$ coefficient values $0,0.5 \%$, $0.75 \%, 1 \%, 5 \%, 10 \%, 25 \%$ by rolling-up level.

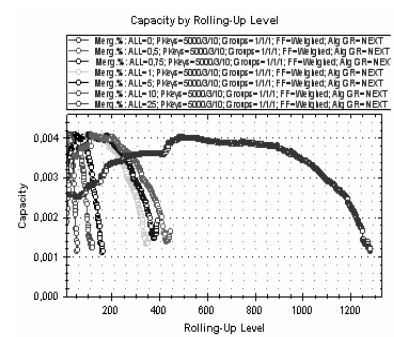

(a)

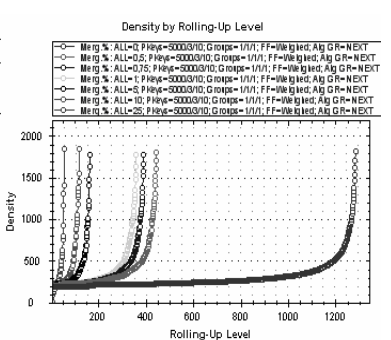

(b)
Fig.10 - Specific capacity and density functions by the rolling-up tree level

\section{STRUCTURAL FEATURES CLUSTERING}

The clustering algorithm was applied for image classification by two merging functions: absolute (1314) and relative (15-16). Within every case there are four different sets of structure features.

As clustering result 8 dendrograms were got [See Fig. 11-14]. Every dendrogram node is connected with database of classified images. User can indicate the node and get full information about images placed on the concrete rolling-up tree level.

Examples of the indicated nodes $(1,2,3,4)$ from dendrogram are presented on Fig. 17-20.

Four sets of the structure features are as follows:

$$
\begin{array}{ll}
\text { A. } & I A, K_{s}^{123}(M C), M(I A), D(I A), R(I A), R_{i}(I A) \\
\text { B. } & I A, K_{s}^{1}, K_{s}^{2}, K_{s}^{3}, K_{s}^{123}(M C), R_{i}(I A) \\
\text { C. } & P X, M C, C, C R, I A, K_{s}^{1}, K_{s}^{2}, K_{s}^{3}, K_{s}^{12}, \\
& K_{s}^{23}, K_{s}^{123}(P X), K_{s}^{123}(M C), M(C R), D(C R), \\
& M(I A), D(I A), R(C R), R(I A), R_{i}(C R), R_{i}(I A) . \\
\text { D. } & C R, I A, R(C R), R(I A), R_{i}(C R), R_{i}(I A) .
\end{array}
$$

Fig.11-14, 17-20 illustrates classification result for 4 semantic groups: dinosaurs, flowers, animals (lions+elaphans), busses.

The image key represents image color scheme $(R$, $G, \quad B$ components), structural characteristics, dimension, etc.

Data information about image key (structure features) was stored in XML file with following structure: 


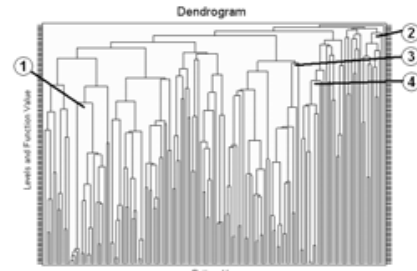

(a)

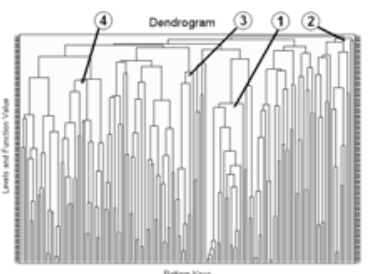

(b)
Fig.11 - Dendrograms for absolute (a) and relative (b) clustering by 6 features from $A$

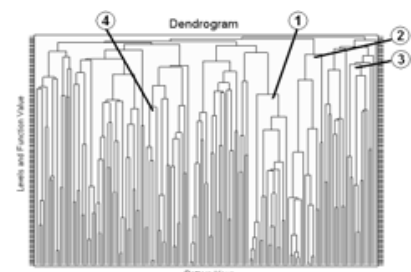

(a)

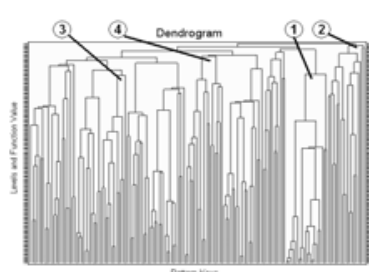

(b)
Fig.12 - Dendrograms for absolute (a) and relative (b) clustering by 6 features from $B$

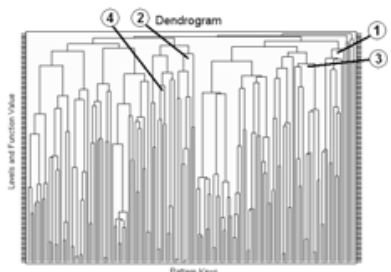

(a)

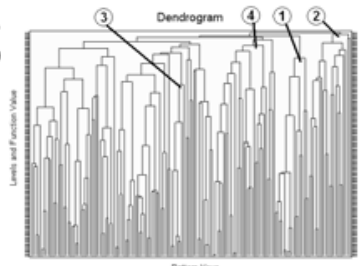

(b)
Fig.13 - Dendrograms for absolute (a) and relative (b) clustering by 20 features from $\mathrm{C}$

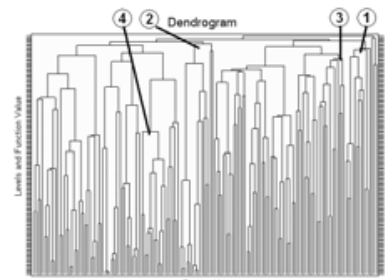

(a)

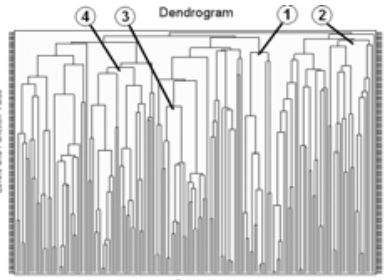

(b)
Fig.14 - Dendrograms for absolute (a) and relative (b) clustering by 6 features from $D$

$<$ ?xml version="1.๑"?>

$<$ Root $>$

<File>D: \IMAGES $\backslash 300 . j p g</ F i l e>$

$<$ Class $>$

$<A>60</ A>$

$<C>30</ C>$

$<\mathrm{D}>10</ \mathrm{D}>$

$</$ Class $>$

$<$ Keys $>$

$<$ Key $>$

$<$ IA $>19</$ IA $>$

$<$ K123MC >0, 0084284</K123MC >

$<$ MIA $>118,736842105263</ M I A>$

$<$ DIA $>130,602991061607</$ DIA $>$

$<$ RIA $>0,909143359888699</$ RIA $>$

$</$ Key $>$

$<$ Key $>$

$</$ Key $>$

. . .

$</$ Keys $>$
$</$ Root $>$

where: Root - is the parent element. The File section presents full file path, including specific database if distributed data store used. The Class section contains number of classified image class names and position (in percent) when fuzzy logic is used, otherwise one class name and position (in \%). The Key section stores information about image keys.

\section{PROGRAM PACKAGE}

The experimental program package with architecture [See Fig.15] and user interface [See Fig. 16] has been developed. It controls all operation stages: dataset input, parameters, intermediate and output clusters, clustering options (full, specified), report of the algorithm work, result parameters, dendrogram.

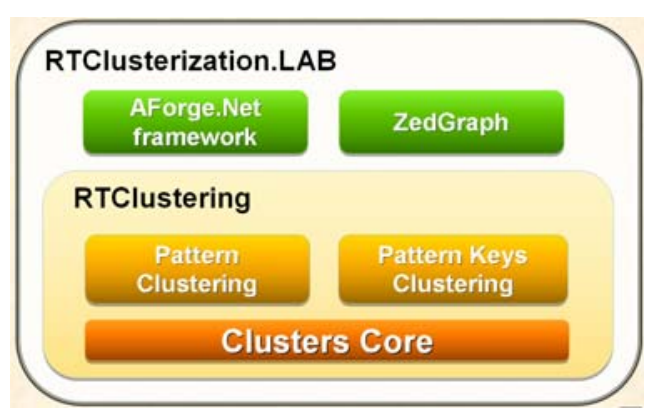

Fig.15 - Program package architecture and component

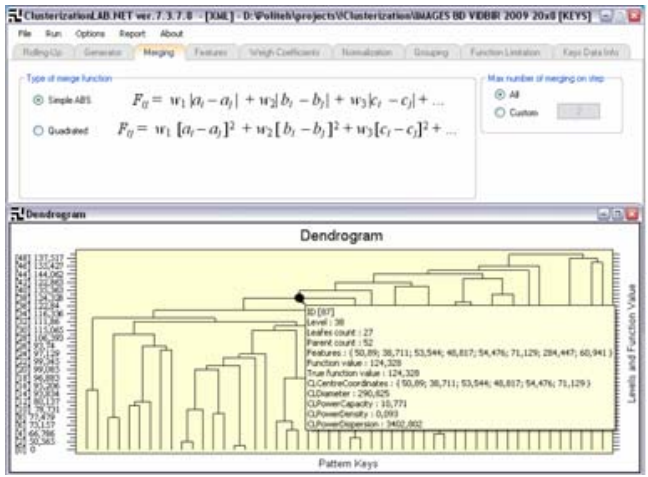

(a)

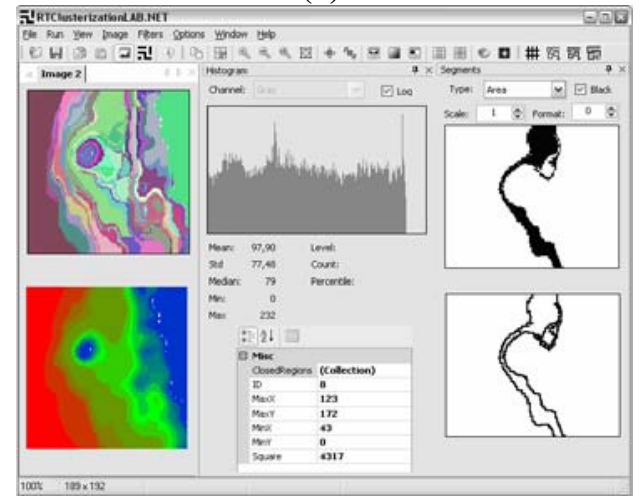

(b)

Fig.16 - Program interfaces 


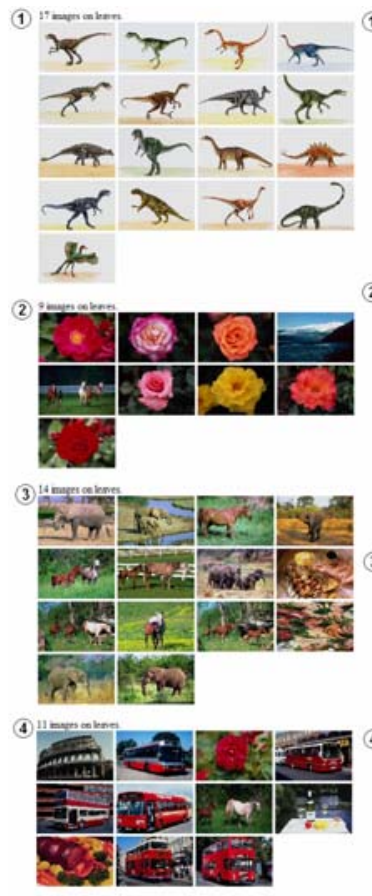

(a)

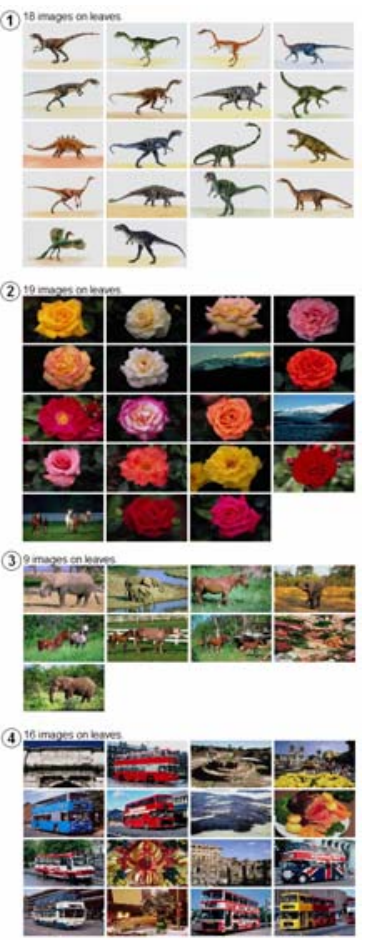

(b)

Fig.17 - Comparing absolute (a) and relative (b) similarity functions for $\mathbf{6}$ features from $A$
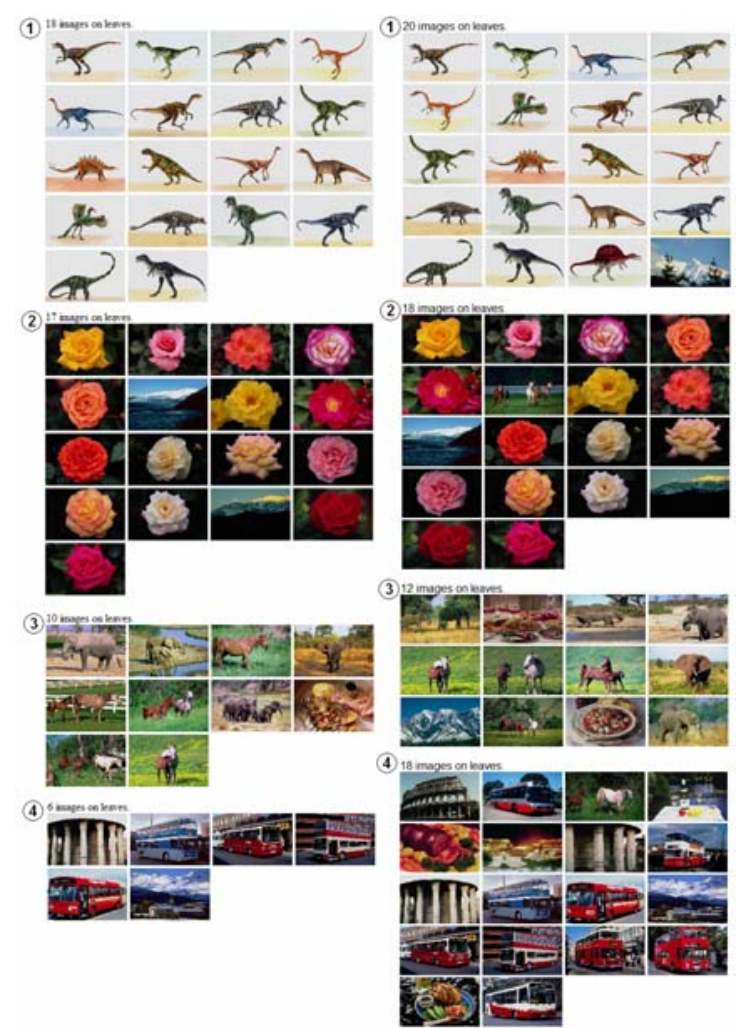

(a)

(b)

Fig.18 - Comparing absolute (a) and relative (b) similarity functions for $\mathbf{6}$ features from $B$

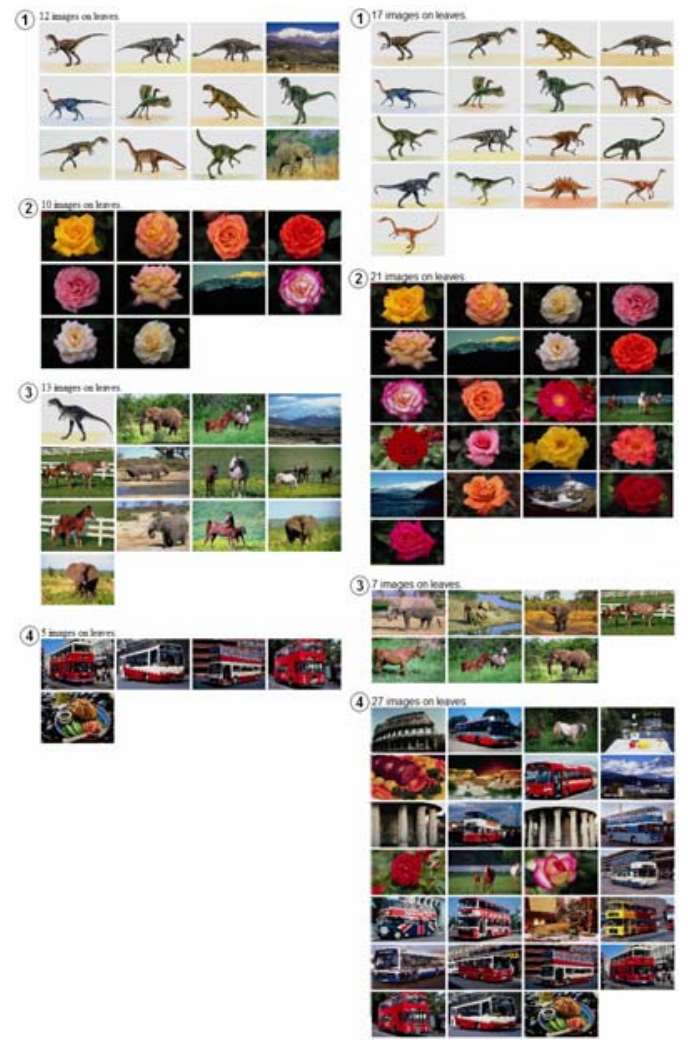

(a)

(b)

Fig.19 - Comparing absolute (a) and relative (b) similarity functions for $\mathbf{2 0}$ features from $\mathbf{C}$

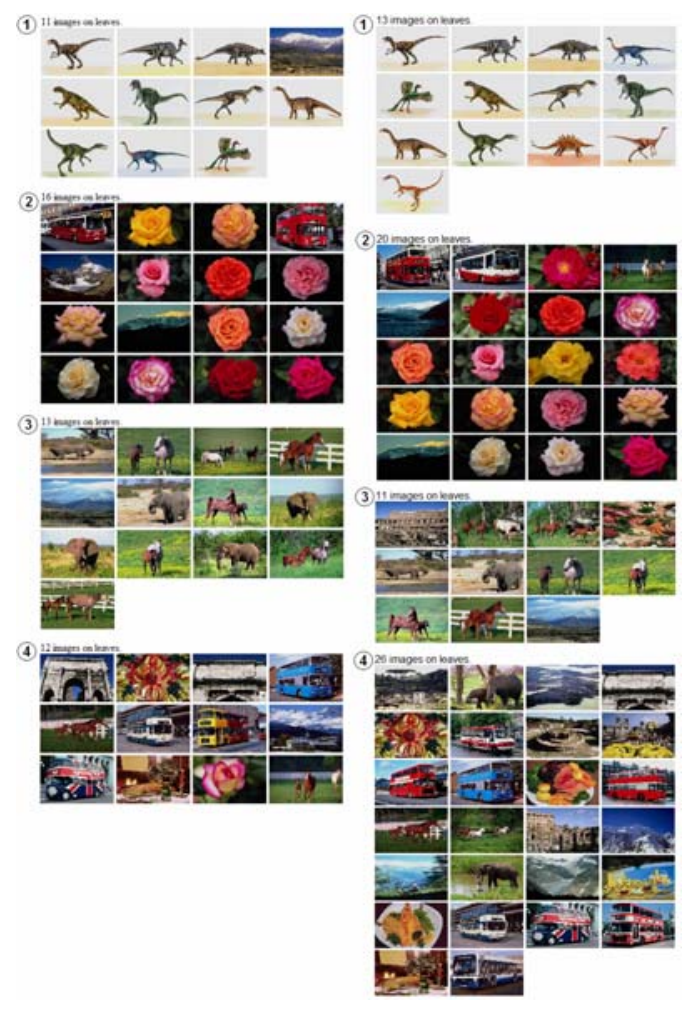

(a)

(b)

Fig.20 - Comparing absolute (a) and relative (b) similarity functions for $\mathbf{6}$ features from $D$ 


\section{CONCLUSION}

The clustering algorithm to get quantitative and qualitative pattern description and relations between them is developed. More than twenty structure features to image classification are proposed.

The agglomerative hierarchical clustering algorithm for data was developed. The new idea to control the algorithm complexity and accuracy by the tolerance and speed coefficient was realized.

Some experiments to image classification by structure features and clustering algorithm were held. They confirmed the algorithm robustness and possibility to control the pattern structure features to get satisfactory classification result as well as the approach application in automated image searching systems.

The program package for data and pattern clustering has been developed. The 3-stages pattern clustering algorithm and tolerance hierarchical data clustering algorithm are main instruments of the package. The package is planned to be applied for large scale data groups including visual pattern, gens or text.

\section{REFERENCES}

[1] A. Vailaya, A. K. Jain, H. J. Zhang, "On image classification: city vs. landscape", Pattern Recognition, vol. 31, p. 1921-1935, 1998.

[2] M. J. Swain, D. H. Ballard, "Color indexing", International journal of Computer Vision, vol. 7, n. 1, p. 11-32, 1991.

[3] H. Nezamabadi-pour, E. Kabir, "Image retrieval using histograms of unicolor and bicolor blocas and direccional changes in intensity gradient", Pattern Recognition Letters, vol. 25, n. 14, p. 1547-1557, 2004.

[4] F. Mokhtarian, S. Abbasi, "Shape similatity retrieval under affine transforms", Pattern Recognition, vol. 35 , p. 31-41, 2002

[5] A. K. Jain, A. Vailaya, "Image retrieval using color and shape", Pattern Recognition, vol. 29, n. 8, p. 1233-1244, 1996.

[6] B. S. Manjunath, W. Y. Ma, "Texture feature for browsing and retrieval of image data", IEEE PAMI, vol. 8, n. 18, p. 837-842, 1996.

[7] J. R. Smith, C. S. Li, "Image classification and quering using composite region templates", Academic Press, Computer Vision and Understanding, vol. 75, p. 165-174, 1999.

[8] J. Z. Wang, J. Li, G. Wiederhold, "SIMPLIcity: semantic sensitive integrated matching for picture libraries", IEEE Trans. on Pattern Analysis and Machine Intelligence, vol. 23, n. 9, p. 947-963, 2001.

[9] H. W. Yoo, S. H. Jung, D. H. Jang, Y. K. Na, "Extraction of major object features using VQ clustering for content-based image retrieval", Pattern Recognition, vol. 35, p. 1115-1126, 2002.

[10] M. Szummer, R. W. Picard, "Indoor-outdoor image classification", IEEE International Workshop on Content-Based Access of Image and Video Database
(ICCV’98), p. 42-51, 1998

[11] T. P. Minka, R. W. Picard, "Interactive learning using a society of models", Pattern Recognition, vol. 30, n. 3, p. 565, 1997.

[12] M. C. Burl, M. Weber, P. Perona, "A probabilistic approach to object recognition using local photometry and global geometry", Proc. European Conf. Computer Vision, p. 628-641, 1998.

[13] J. Z. Wang, M. A. Fishler, "Visual similarity, judgmental certainty and stereo correspondence", Proc. DARPA Image Understanding Workshop, 1998.

[14] R. Melnyk, R. Tushnytskyy, "Decomposition of Visual Patterns", Proc. of $9^{\text {th }}$ Intern. Conf. CADSM2007, p. 278-279, 2007.

[15] R. Melnyk, R. Tushnytskyy, "Image Classification by

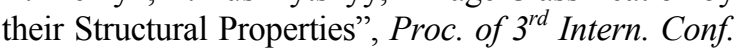
CSIT-2008, p. 37-40, 2008.

[16] R. Melnyk, R. Tushnytskyy, "Cluster Parameters Control in Pattern Decomposition Algorithm", Information Extraction and Processing. - 2007. - № 27 (103). - P. 58-62. - (in ukrainian)

[17] Andy M. Yip, Chris Ding, Tony F. Chan. Dynamic Cluster Formation Using Level Set Methods. - IEEE Trans. on Pattern Analysis and Machine Intelligence, vol.28, n. 6, pp.877-889, June, 2006.

[18] Leo Grady, Eric L. Schwartz. Isoperimetric Graph partitioning for Image segmentation. - IEEE Trans. on Pattern Analysis and Machine Intelligence, vol.28, n. 3, pp.469-475, March, 2006.

[19] M. Pavan, M. Pelillo. Dominant sets and Pairwise Clustering. - IEEE Trans. on Pattern Analysis and Machine Intelligence, vol.29, n. 1, pp.167-172, January, 2007.

[20] C. Ding, X. He. Cluster Aggregate Inequality and Multilevel Hierarchical Clustering, Proc. 9th European Conf. Principles of Data Mining and Knowledge Discovery (2005). p. 71-83.

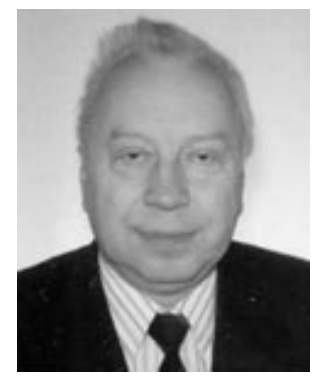

Roman Melnyk. Professor of Software Engineering Department, Institute of Computer Sciences and Information Technologies, Lviv Polytechnic National University.

Research interests: combinatory problems, methods of fuzzy clustering, coding and recognition, storage and data mining.

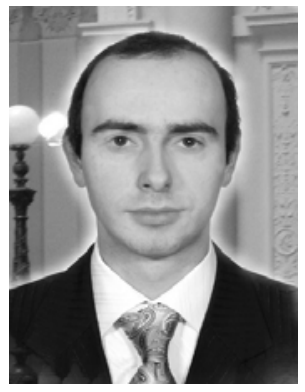

Ruslan Tushnytskyy. A postgraduate student at the Software Engineering Department, Institute of Computer Sciences and Information Technologies, Lviv Polytechnic National University.

Research interests: preliminary processing of images, coding and recognition in real time, clustering algorithms, data compression. 